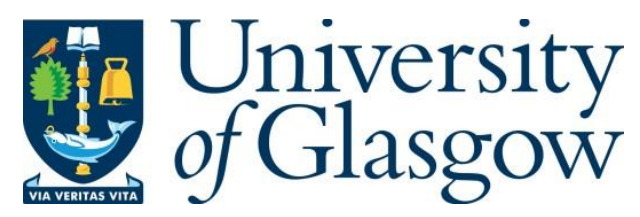

Zhang, X., Zhang, L., Xiao, P. and Wei, J. (2019) Fine timing synchronization based on modified expectation maximization clustering algorithm for OFDM systems. IEEE Wireless Communications Letters, 8(5), pp. 1452-1455.

There may be differences between this version and the published version. You are advised to consult the publisher's version if you wish to cite from it.

http://eprints.gla.ac.uk/187660/

Deposited on: 5 July 2019

Enlighten - Research publications by members of the University of Glasgow http://eprints.gla.ac.uk 


\title{
Fine Timing Synchronization Based on Modified Expectation Maximization Clustering Algorithm for OFDM Systems
}

\author{
Xiaoying Zhang, Lei Zhang, Pei Xiao, Senior Member, IEEE, Jibo Wei
}

\begin{abstract}
A novel fine timing synchronizaton method based on the modified expectation-maximization (EM) clustering algorithm is proposed for OFDM systems. Using the cross-correlation metrics of one preamble symbol, the cross-correlation peaks corresponding to the channel arriving paths are identified by the proposed modified EM clustering algorithm, the position of the first coherent cross-correlation peak is then chosen as the start of the frame. Computer simulations show that the proposed method is robust in multipath dispersive channels and achieves superior performance to existing techniques in terms of timing accuracy.
\end{abstract}

Index Terms-Orthogonal frequency-division multiplexing (OFDM), synchronization, timing offset, expectation maximization

\section{INTRODUCTION}

Orthogonal Frequency Division Multiplexing (OFDM) is an important multicarrier technique for modern wireless communication systems. It has been widely adopted in broadband wireless communications standards, such as Long Term Evolution (LTE) and IEEE 802.11, etc. In addition, it has been recently identified as the waveform for the fifth-generation (5G) wireless communication systems. In order to keep orthogonality among subcarriers in the OFDM system, the transmitter and receiver should be well synchronized in the time domain [1]-[4]. Timing offset due to the imperfect symchronization may introduce inter-symbol interference (ISI) and inter-carrier interference (ICI), which can severely degrade the system performance.

Among the existing timing synchronization methods in the literature, preamble-aided methods yield robust performance with high efficiency and therefore have been widely applied to the burst packet transmission systems [5]. In the frequency selective fading channels, the accurate timing position of the OFDM frame is the instant corresponding to the arrival of the preamble via the first channel path [6]-[9]. However, the first channel path may not be the strongest one, which makes the accurate timing detection challenging. In many existing timing synchronization methods, the position of maximum timing metrics related to the strongest arriving path is taken

This work was supported in part by the Research Found of National University of Defense Technology under Grant ZK17-03-13. X. Zhang and J. Wei are with the College of Electronic Science and Technology, National University of Defense Technology, China (e-mail: zhangxiaoying@nudt.edu.cn; wjbhw@nudt.edu.cn).

L. Zhang is with the Shchool of Engineering, University of Glasgow, U.K. (e-mail: Lei.Zhang@glasgow.ac.uk).

P. Xiao is with the Institute for Communication Systems (ICS), University of Surrey, U.K. (e-mail: P.xiao@surrey.ac.uk). as the frame start position instead [1]-[5]. The well-known Schmidl and Cox [1] algorithm used the auto-correlation of a preamble symbol with two identical parts to estimate timing position. This method is robust to carrier frequency offset (CFO) but suffers from the timing metric plateau. The new preamble structures were designed by Minn [2], Park [3] and Ren [4] to make the auto-correlation type timing metrics sharper. However, these methods have large timing estimation variance for multipath channels. A smoothed cross-correlation metrics based scheme was proposed in [5] to accomplish timing synchronization. All of the aforementioned methods can only find the position of the maximum timing metric peak; hence, they may inherently induce a later timing offset when there exists a relatively large time difference between the first path and the strongest path. If no appropriate forward-shift timing protection is used, they may suffer from both ISI and ICI. In order to search for the timing position corresponding to the first arriving channel path, a threshold was first calculated in [6] based on the statistical properties of timing metrics and the preset probability of false detection, then the position of the first peak above the threshold was detected as the timing position. This method was adopted and further extended in [7] and [8]. However, the performance of these thresholdbased methods depends on the noise variance estimation and the accurate analysis of statistical properties, mismatch may happen between the expectations and practical performance [9].

To effectively identify the frame start position that corresponds to the arrival of the preamble via the first channel path, a novel fine timing synchronization scheme is proposed in this paper. Unlike the existing statistical threshold-based detection methods, the proposed scheme presents the modified expectation-maximization (EM) clustering algorithm to identify the coherent strong Gaussian distributed cross-correlation metrics corresponding to the path timing points and the weak Gaussian metrics. The position of the first coherent metric is taken as the frame start position. To the best knowledge of the authors, this is the first time that the clustering-type algorithm is adopted to solve the timing synchronization problem. The simulation results indicate the proposed method provides superior timing accuracy performance compared with the previous schemes over the multipath channels. In the rest of the paper, we use $\mathcal{C N}\left(m, v^{2}\right)$ to denote the circular Gaussian distribution with mean $m$ and variance $v^{2}$, and the corresponding probabilty density function (PDF) for random variable $x$ is represented by $\mathcal{C N}\left(x ; m, v^{2}\right) \triangleq\left(\pi v^{2}\right)^{-1} \exp \left(-|x-m|^{2} / v^{2}\right)$. 


\section{SYSTEM MODEL AND COARSE SYNCHRONIZATION}

In this section, we first describe an OFDM system and then address the coarse timing and frequency synchronization procedure which serve as a preprocessing of the proposed fine timing scheme. Let us consider an OFDM system with fast Fourier transform (FFT) size $N$ and cyclic prefix (CP) length $L_{C P}$. The $N_{\text {use }}\left(N_{u s e} \leq N\right)$ useful subcarriers are used to transmit the data symbols while the $N-N_{u s e}$ virtual subcarriers at the edges of the spectrum are used as guard bands. One OFDM symbol length is $N_{s}=N+L_{C P}$. The time domain transmitted signal can be written as

$$
x(n)=\frac{1}{\sqrt{N}} \sum_{k=N_{u s e} / 2}^{N_{u s e} / 2-1} X(k) e^{j \frac{2 \pi}{N} k\left(n-L_{C P}\right)}, 0 \leq n \leq N_{s},
$$

where $X(k)$ is the frequency-domain transmitted signal. Assume that the discrete-time multipath fading channel has path gains $\{h(l)\}_{l=0}^{L-1}$ where $L$ is the maximum delay. The received signal can be expressed as

$$
r(n)=\sum_{l=0}^{L-1} h(l) x(n-\theta-l) e^{j \frac{2 \pi}{N} \varepsilon n}+w(n),
$$

where $\theta$ is the timing offset in samples and $\varepsilon$ is the $\mathrm{CFO}$ normalized to the subcarrier spacing. $w(n)$ denotes the zeromean additive white Gaussian noise (AWGN). Before the fine timing synchronization, coarse timing estimation, $\mathrm{CFO}$ estimation and correction are performed. Consider a typical preamble structure which consists of a $\mathrm{CP}$ part $A_{C P}$ and two repetitive sequences $[A A]$, i.e., $S=\left[\begin{array}{lll}A_{C P} & A & A\end{array}\right]$, where $A$ is the time-domain random sequence with length $N / 2$. The coarse timing position $T_{c}$ is estimated by maximizing the following timing metric as [1]:

$$
T_{c}=\arg \max _{d}\left\{\frac{1}{L_{C P}+1} \sum_{k=0}^{L_{C P}} M(d-k)\right\}
$$

where

$$
\begin{gathered}
M(d)=\frac{|P(d)|^{2}}{\sum_{k=0}^{N-1}|r(d+k)|^{2}}, \\
P(d)=\sum_{k=0}^{N / 2-1} r^{*}(d+k) r\left(d+k+\frac{N}{2}\right) .
\end{gathered}
$$

In (4), $M(d)$ represents the energy ratio between $P(d)$ and the received signal. An integrator with length $L_{C P}+1$ is applied to $M(d)$ in (3) to reduce the plateau effect [1].

Besides the timing synchronization, the carrier-frequency synchronization is another important task in practical systems. A large CFO may make the timing metric peaks ambiguous and thus deteriorate the timing performance. Here, we focus on the symbol timing synchronization and suppose CFO can be estimated and totally compensated by the existing algorithm, e.g., [1], [10]. Let the CFO corrected received signal be $\hat{r}(n)$. After the coarse synchronization procedures, $\hat{r}(n)$ and $T_{c}$ are fed to the fine timing synchronization.

\section{Proposed Fine Timing Synchronization}

The proposed fine timing scheme consists of two steps. First, we use cross-correlation timing metrics to determine the position of the strongest channel path. Second, the crosscorrelation metrics within a restricted clustering range are classified by the modified EM algorithm into the coherent part corresponding to path timing points and non-coherent part, and finally, the first path timing position is identified as the beginning of the frame.

First Step: Let $T_{s}$ denote the timing position corresponding to the strongest path, it can be searched within a limited range around the coarse timing position $T_{c}$ as follows

$$
\begin{gathered}
T_{s}=\arg \max _{d}\left(\left|P_{c}(d)\right|\right), d \in\left[T_{c}-N / 2, T_{c}+N / 2\right] \\
P_{c}(d)=\sum_{k=0}^{N_{s}-1} \hat{r}(d+k) S^{*}(k) .
\end{gathered}
$$

where $S(k), 0 \leq k \leq N_{s}-1$, denote the $k$ th element of preamble symbol $S . P_{c}(d)$ is the cross-correlation timing metric between the frequency corrected received signal and the known preamble.

Second Step: In this step, the EM algorithm is used to identify the first channel path timing point. We first describe the clustering features of the cross-correlation timing metrics and show that the path timing detection can be approximately regarded as a binary classification problem in the Gaussian mixture model, and then introduce the proposed modified EM cluttering algorithm. Based on (7), the cross-correlation metric can be written as [7]

$$
\begin{aligned}
P_{c}(d) & =h(d-\theta) \sum_{k=0}^{N_{s}-1}|S(k)|^{2} \\
& +\sum_{k=0}^{N_{s}-1}\left(\sum_{l \neq d-\theta} h(l) S(d+k-\theta-l)+w(d+k)\right) S^{*}(k),
\end{aligned}
$$

where $\sum_{k=0}^{N_{s}-1}|S(k)|^{2}=E_{s}$ is the energy of preamble and $h(l)$ is a zero-mean complex Gaussian variable with variance $\nu_{l}^{2}$. The first term of (8) is the coherent part which comes from a full-preamble pattern match between the arrived preamble via a channel path and its known version [7]. This term follows complex Gaussian distribution with zeromean and variance $E_{s}^{2} \nu_{l}^{2}, l=d-\theta$. The second term of (8) is the random part, which is the summation of many random terms and can be approximated as a complex Gaussian variable with zero mean and relatively smaller variance $\sigma_{e}^{2}$ using the central limit theorem. For the path timing position $d \in\{\theta, \theta+1, \cdots, \theta+L-1\}, P_{c}(d)$ can be approximated as a zero-mean complex Gaussian variable with large variance $\sigma_{0}^{2} \approx E_{s}^{2} \nu_{l}^{2}+\sigma_{e}^{2}$. When $d \notin\{\theta, \theta+1, \cdots, \theta+L-1\}$, the first term in (8) vanishes, $P_{c}(d)$ degenerates to be a zero-mean Gaussian variable with small variance $\sigma_{1}^{2}=\sigma_{e}^{2}$. To sum up, the cross-correlation metrics $P_{c}(d)$ can be divided into two clusters labeled by the latent state $C_{d} \in\{0,1\}$. One is the strong Gaussian cluster with $C_{d}=0$, where $d$ corresponds to the path timing position and $P_{c}(d) \sim \mathcal{C N}\left(0, \sigma_{0}^{2}\right)$. The other is the weak Gaussian cluster with $C_{d}=1$, where $d$ is not related to any effective channel arriving path and $P_{c}(d) \sim \mathcal{C N}\left(0, \sigma_{1}^{2}\right)$. Note that although we neglect the influence of fluctuation of 
different channel path power $\nu_{l}^{2}, 0 \leq l \leq L-1$, only the major channel paths with significant $E_{s}^{2} \nu_{l}^{2}$ are considered as effective coherent timing paths. The strong clusters and the weak clusters can be identified by the EM algorithm [11]. Fig. 1 shows an example of cross-correlation metrics and the classification result after applying the proposed algorithm. Here, COST 207 rural area (RA) channel model [12] with 4 paths is considered. Although on average, the first path of RA channel has the maximum power, there are approximate $27 \%$ of channel samples whose first path is not the strongest one in the simulation. It can be seen that the cross-correlation metrics are divided into the weak Gaussian cluster with $C_{d}=1$ and the strong coherent cluster with $C_{d}=0$. The coherent cluster contains timing points corresponding to the major channel paths, e.g., from the first to the third path of the channel. The fourth path in the channel tail has a very small $\nu_{l}^{2}$ and, thus, its cross-correlation metrics can not be distinguished from the non-coherent part.

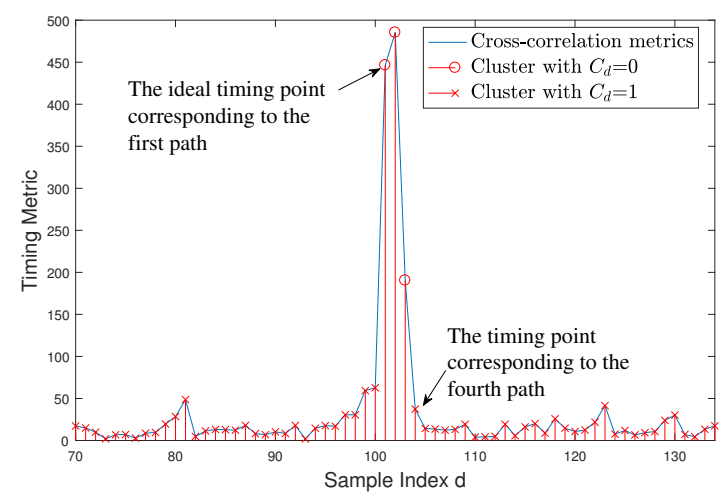

Fig. 1. Timing metric and classification results for COST 207 RA channel.

Considering $U$ cross-correlation timing metrics around the strongest path position $T_{s}$ as the clustering range, $P_{c}(d)$ belonging to the range $\mathcal{J}=\left[T_{s}-U / 2, T_{s}+U / 2\right]$ are clustered by the EM algorithm. The parameters set of EM clustering algorithm is $\left\{P_{0}, P_{1}, \sigma_{0}^{2}, \sigma_{1}^{2}\right\}$, where $P_{0}$ denotes the a priori probability of the cross-correlation metrics following the strong Gaussian distribution $\mathcal{C N}\left(0, \sigma_{0}^{2}\right)$, and $P_{1}$ denotes the a priori probability of the cross-correlation metrics following the weak Gaussian distribution $\mathcal{C N}\left(0, \sigma_{1}^{2}\right)$. The proposed EM clustering algorithm iterates as follows. In the E step of $i$ th iteration, the responsibility $\omega_{d}^{(i)}(k)$ that cluster $k$ takes for metric $P_{c}(d)$ is updated as

$$
\omega_{d}^{(i)}(k)=\frac{P_{k}^{(i-1)} \cdot \mathcal{C N}\left(P_{c}(d) ; 0, \sigma_{k}^{2}(i-1)\right)}{\sum_{k=0}^{1} P_{k}^{(i-1)} \cdot \mathcal{C N}\left(P_{c}(d) ; 0, \sigma_{k}^{2}(i-1)\right)}, k=0,1
$$

where $P_{k}^{(i-1)}$ and $\sigma_{k}^{2}(i-1)$ represent the a priori probability and variance for cluster $k$ in the $(i-1)$ th iteration, respectively. Then they are updated in the M step as follows

$$
\begin{aligned}
\sigma_{k}^{2}(i) & =\frac{\sum_{d} \omega_{d}^{(i)}(k)\left|P_{c}(d)\right|^{2}}{\sum_{d} \omega_{d}^{(i)}(k)}, k=0,1 \\
P_{0}^{(i)} & =\min \left\{\sum_{d} \omega_{d}^{(i)}(0) / U, c / U\right\},
\end{aligned}
$$

$$
P_{1}^{(i)}=1-P_{0}^{(i)}
$$

Compared with the traditional EM algorithm [11], updating function of $P_{0}^{(i)}$ is modified in the proposed scheme. As shown in (11), we set the priori probability of strong Gaussian cluster $P_{0}^{(i)}$ less than a preset small constant $c / U$. This limitation comes from two inferences: first, for sparse multipath channel, the number of the coherent cross-correlation peaks is much less than that of the non-coherent metrics among the clustering range, which naturally lead to a small $P_{0}^{(i)}$. On the other hand, we find that the limiting $P_{0}^{(i)}$ being a under-estimated small value can avoid the EM clustering algorithm taking the fake cross-correlation peaks as the coherent peaks, which greatly improve the timing accuracy. To validate this modification, the impact of parameter $c$ is evaluated by simulations under the different channels. The value of $U$ is set to $L_{C P}$. As depicted in Fig. 2 and Fig. 3, mean square error (MSE) of timing offset for the proposed algorithm is shown with different values of $c$ and signal-to-noise ratios (SNR) for COST 207 RA and typical urban (TU) channel models [12], respectively. When $c=U$, the modified EM clustering algorithm turns to the conventional EM algorithm, which have relatively poor performance in both cases. It is observed that when $c=1 \sim 2$, the proposed EM algorithm achieved robust performance with timing MSE less than 1 for the whole SNR ranges in both RA and TU channels.

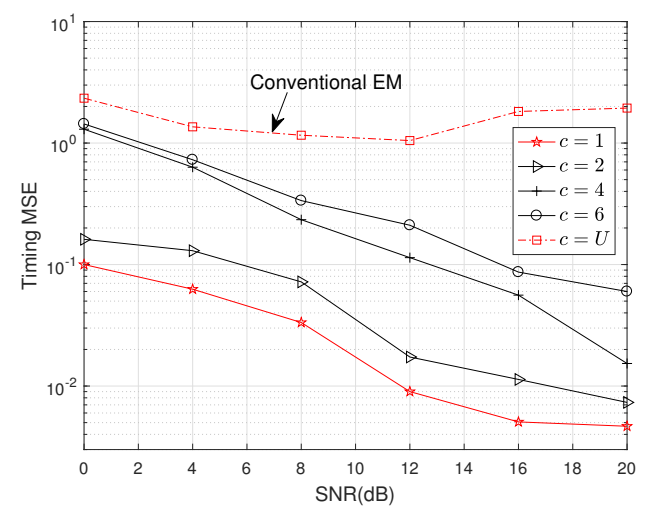

Fig. 2. Timing performance for different values of $c$ for COST 207 RA channel.

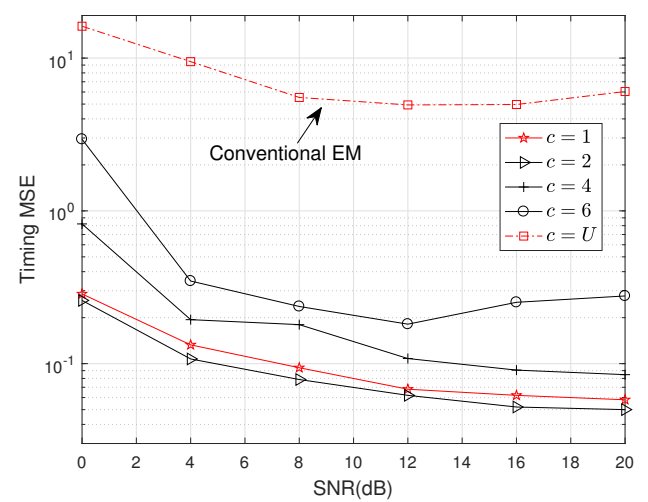

Fig. 3. Timing performance for different values of $c$ for COST $207 \mathrm{TU}$ channel.

For the first iteration, we set $P_{0}^{(0)}=c / U, P_{1}^{(0)}=1-P_{0}^{(0)}$, $\sigma_{0}^{2}(0)=\max \left(\left|P_{c}(d)\right|^{2}\right)$ with $d \in \mathcal{J}$ and $\sigma_{1}^{2}(0)=\sigma_{0}^{2}(0) / 10$. The Log likelihood $L^{(i)}$ is calculated for each iteration as 


$$
L^{(i)}=\sum_{d=T_{s}-U / 2}^{T_{s}+U / 2} \log \left(\sum_{k=0}^{1} P_{k}^{(i)} \cdot \mathcal{C N}\left(P_{c}(d), 0, \sigma_{k}^{2}(i)\right)\right)
$$

When $\left|L^{(i)}-L^{(i-1)}\right|$ is less than a small value, e.g., 0.01 , the iterations are stopped and the latent state $C_{d} \in\{0,1\}$ for $P_{c}(d)$ can be calculated as

$$
C_{d}=\arg \max _{k}\left\{P_{k}^{(i)} \cdot \mathcal{C N}\left(P_{c}(d), 0, \sigma_{k}^{2}(i)\right)\right\}, k=0,1
$$

The smallest $d$ with $C_{d}=0$ is chosen as the fine timing position.

\section{Simulation Results}

Computer simulations are carried out to evaluate the performance of the proposed fine timing method compared with other existing methods given by [1], [4]-[6], [9]. We consider an OFDM system with $N=512, N_{u s e}=450, L_{C P}=64$ and a bandwidth of $4 \mathrm{MHz}$. The normalized CFO is set as $\varepsilon=0.1$ and it is estimated and corrected by the algorithm in [1] before the fine timing procedure. The preamble with two identical parts is generated by mapping the Zadoff Chu sequence [13] with index 23 to the even numbered subcarriers [1]. We set the length of clustering range $U=L_{C P}$ and $c=1$. When the difference of the Log likelihood between adjacent iterations is smaller than 0.01, the EM iterations are stopped. In our simulations, the stopping criterion can be satisfied within 6 iterations. Fig. 4 compares the MSE of the timing offset for different algorithms for the COST 207 RA channel model. Fig. 5 compares the MSE of the timing offset for the proposed method with the previous methods under the COST $207 \mathrm{TU}$ channel model, which has 29 taps in the underlying system parameters setting.

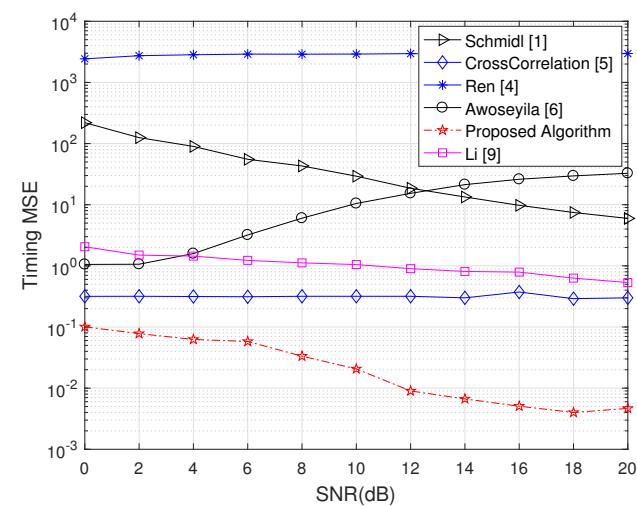

Fig. 4. Timing MSE of different algorithms in the COST 207 RA Channel.

As shown in both Fig. 4 and Fig. 5, the proposed method achieves superior performance to the existing methods in both channels. As mentioned in [8], the timing MSE in [6] increases with the SNR due to the fact that the threshold-based algorithm suffers from fake peaks before the first path. The proposed algorithm outperforms the method in [9] because the differential cross-correlation timing metrics proposed in [9] incorporate extra terms compared to the cross-correlation metrics, which may make the first path timing point ambiguous. The timing estimates in [5] tend to search strongest arriving path and thus its performance is worse than the proposed scheme.

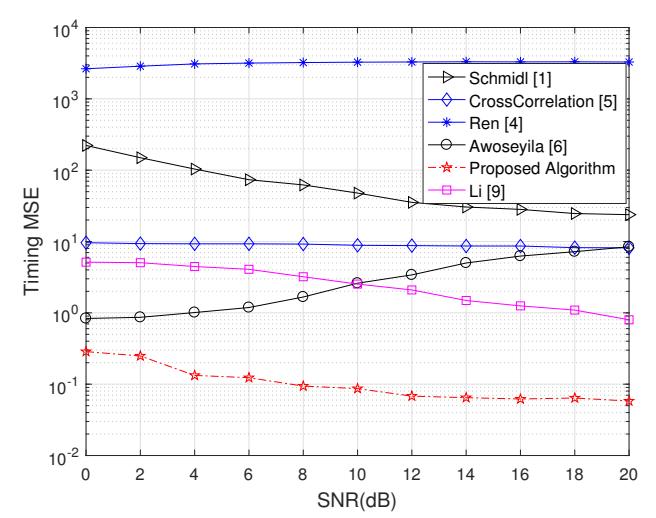

Fig. 5. Timing MSE of different algorithms in the COST $207 \mathrm{TU}$ channel.

\section{CONCLUSION}

A novel fine timing synchronization method for OFDM systems is presented in this paper. By using the modified EM clustering algorithm, the proposed method classifies the fine timing metrics as the coherent strong Gaussian distributed cluster and the weak Gaussian cluster, the position of the first coherent metrics is taken as the start of the frame. Simulation results show that the proposed algorithm has superior performance compared with the existing methods. The proposed scheme is widely applicable to other practical wireless communication systems, e.g., TDS-OFDM or CDMA systems.

\section{REFERENCES}

[1] T. M. Schmidl and D. C. Cox, "Robust frequency and timing synchronization for OFDM," IEEE Trans. Commun., vol. 45, no. 12, pp. 1613-1621, Dec. 1997.

[2] H. Minn, M. Zeng, and V. K. Bhargava, "On timing offset estimation for OFDM systems," IEEE Commun. Lett., vol. 4, no. 7, pp. 242-244, Jul. 2000.

[3] B. Park, H. Cheon, C. Kang, and D. Hong, "A novel timing estimation method for OFDM systems," IEEE Commun. Lett., vol. 7, no. 5, pp. 239-241, May 2003.

[4] G. Ren, Y. Chang, H. Zhang, and H. Zhang, "Synchronization methods based on a new constant envelope preamble for OFDM systems," IEEE Trans. Broadcast., vol. 51, no. 1, pp. 139-143, Mar. 2005.

[5] R. van Nee and R. Prasad, OFDM for Wireless Multimedia Communications. Norwell, MA: Artech House, 2000, pp. 86-91.

[6] A. B. Awoseyila, C. Kasparis, and B. G. Evans, "Improved preamble aided timing estimation for OFDM systems," IEEE Commun. Lett., vol. 12, no. 11, pp. 825-827, Nov. 2008.

[7] A. B. Awoseyila, C. Kasparis, and B. G. Evans, "Robust time-domain timing and frequency synchronization for OFDM systems," IEEE Trans. Consum. Electron., vol. 55, no. 2, pp. 391-399, May 2009.

[8] H. Abdzadeh-Ziabari and M. G. Shayesteh, "Robust timing and frequency synchronization for OFDM systems", IEEE Trans. Veh. Technol., vol. 60 , no. 8, pp. 3646-3656, Oct. 2011.

[9] L. Zhen, H. Qin, B. Song, and R. Ding, "Frame timing estimation based on statistical analysis for orthogonal frequency division multiplexing systems in multipath fading channels," IET Commun., vol. 10, no. 16, pp. 2125-2134, 2016.

[10] Y. H. Kim, I. Song, S. Yoon, and S. Park, "An efficient frequency offset estimator for OFDM systems and its performance characteristics," Trans. Veh. Technol., vol. 50, no. 5, pp. 1307-1312, Sept. 2001.

[11] C. M. Bishop, Pattern recognition and machine learning. New York: springer, 2006, pp. 435-443.

[12] P. Hoeher, "A statistical discrete time model for the WSSUS multipath channel", IEEE Trans. Veh. Technol., vol. 41, no. 4, pp. 461-468, Nov. 1992.

[13] D. C. Chu, "Polyphase codes with good periodic correlation properties," IEEE Trans. Inf. Theory, vol. 18, no.4, pp.531-532, Jul. 1972. 